\title{
Neisseria elongata subsp. glycolytica subsp. nov.
}

\section{S. D. HENRIKSEN AND EIRIK HOLTEN}

Kaptein W. Wilhelmsen og Frues Bakteriologiske Institutt, University of Oslo, Rikshospitalet, Oslo 1, Norway

A rod-shaped, saccharolytic strain of Neisseria isolated from the throat of a patient with pharyngitis and considered to represent a new subspecies of Neisseria elongata is described. The strain differs from $N$. elongata subsp. elongata in producing acid from glucose, in giving a strong catalase reaction, and in the consistency of colonies on agar media. The name $N$. elongata subsp. glycolytica is proposed for the new subspecies, of which strain 6171/75 (= ATCC $29315=$ NCTC 11050) is the type.

The purpose of this paper is to name and describe a rod-shaped strain of Neisseria isolated from the human pharynx and identified as belonging to a new subspecies of Neisseria elongata.

\section{MATERIALS AND METHODS}

Bacterial strains. Strain $6171 / 75$ was isolated from the throat of a 22-year-old woman suffering from acute pharyngitis. A culture of a nose swab from this patient was sterile. The throat culture did not contain known pathogens, but it did contain a mixture of bacteria thought to be commensals, including a strain designated as 6171/75.

In addition to strain $6171 / 75, N$. meningitidis $\mathrm{M} 1$ (12), $N$. elongata M2, $N$. catarrhalis $\mathrm{Ne} 11$, and Moraxella nonliquefaciens $4663 / 62$ were used in this study.

Methods. The methods used were mainly those described previously by Bøvre and Henriksen (5).

Transformation of streptomycin resistance was performed as described by Lie (13). Deoxyribonucleic acid (DNA) was prepared by the method of Bøvre (2). The DNA solution was treated with ribonuclease and Pronase before the final deproteinization. During transformation, log-phase cells were exposed to DNA (10 $\mu \mathrm{g} / \mathrm{ml})$ for $30 \mathrm{~min}$ and were then treated with deoxyribonuclease and plated after proper dilution.

Antibiotic susceptibility tests were carried out by means of filter-paper disks manufactured by $\mathrm{A} / \mathrm{B}$ Biodisk, Stockholm, according to the directions given by the manufacturer.

\section{RESULTS}

Description of strain 6171/75: Micromorphology. Very small, short, slender rods with a marked tendency to occur in pairs; gram negative; nonmotile; very similar to the cells of $N$. elongata $(4,6)$.

Colonies. In primary culture on $5 \%$ human blood agar, the colonies were relatively large ( 2 to $3 \mathrm{~mm}$ in diameter after about $20 \mathrm{~h}$ of incubation), gray, opaque, moderately raised with a flat top, and smooth with a soft, homogeneous consistency. The colonies were easily emulsi- fied in saline. There was no hemolysis on human blood agar. After a few days, the colonies appeared to have a slight yellowish tinge on $5 \%$ human blood agar.

In the first subculture (Fig. 1) on the same medium, as well as in later subcultures, but primarily in the first few transfers, four different colony types appeared. Type 1 was a relatively large, rather flat colony, which after 3 to 4 days developed a slightly irregular surface and margin (Fig. 2). Under the colony, there was distinct "corrosion" (Fig. 3) with a narrow furrow corresponding to the margin of the colony and a small central pit (9). After about 1 week, tiny papillae often appeared on top of these colonies. Type 2 was a round, convex, nearly hemispherical smooth colony with a shiny surface, about 1 to $2 \mathrm{~mm}$ in diameter after $24 \mathrm{~h}$ (Fig. 4). When the colonies were gently removed, a barely visible mark was seen of the agar surface, but there was no "corrosion." Type 3 was a colony similar to the second type, but with low, tongue-like processes from one or both sides or from around the margin of the colony (Fig. 1). Subcultures of this type of colony produced mixtures similar to those seen in the first subculture. Type 4 was a flat, smooth colony with a slightly irregular margin (Fig. 5) that was later derived from the type 2 colony.

Subcultures of types 1,2 , and 4 tended to become more stable in subculture, but there still remained a tendency to variation, particularly of type 1 towards type 2 .

We looked for signs of surface spreading of the colonies (as seen in the spreading-corroding [SC] type of colony of certain Moraxella species $[3,8,10]$ and in $N$. elongata [4]) in numerous cultures incubated in a humid atmosphere for 1 week or more but, with one exception, with negative results. The exception was a bloodagar culture of colony type 1 , where thin, granular zones closely resembling those seen around colonies of some Moraxella species $(8,10)$ and of 


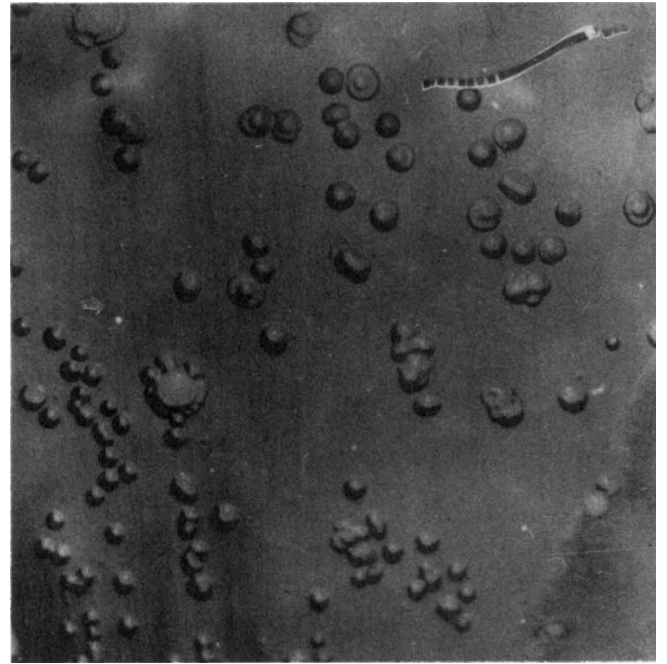

Fig. 1. Blood agar, $48 h \times 4$. One large irregular colony (type 1), many round smooth colonies (type 2), several smooth colonies with offshoots. From 1 st subculture of strain 6171/75. Magnification, $\times 4$.

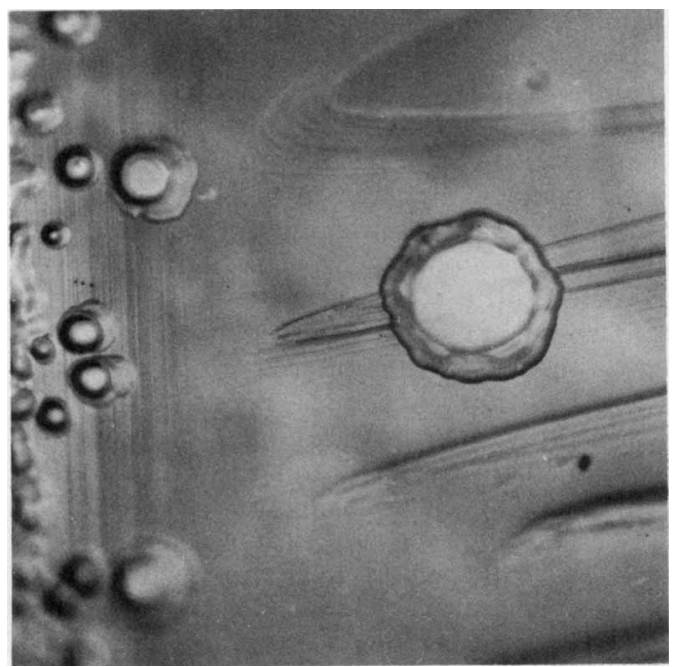

FIG. 2. Blood agar, 4 days. Large colony with irregular margin (type 1). Magnification, $\times 4$.

$N$. elongata (4) appeared around some of the colonies (Fig. 6), mostly colonies growing close to a contaminating Staphylococcus or Micrococcus colony. A stimulating effect of contaminating colonies on this kind of surface spread has previously been noted in other spreading bacteria (11). Similar zones have so far not been observed in cultures of colony types 2 and 4 .

Physiology. The strain was a strict aerobe. Growth occurred at $30^{\circ} \mathrm{C}$ (poor), but not at $20^{\circ} \mathrm{C}$, was only slightly improved by a humid atmos- phere, and was not further improved by the addition of $\mathrm{CO}_{2}$ to the atmosphere. The strain stayed viable for at least 10 days at $35^{\circ} \mathrm{C}$ in a humid atmosphere. It appeared to be somewhat fastidious and showed rather poor growth in most fluid media but reasonably good growth on nutrient agar slants.

Biochemical reactions. A weakly acid reaction, due to oxidation, was produced from glucose in the course of several days. No acid was produced from galactose, fructose, xylose, rhamnose, mannose, maltose, sucrose, or lac-

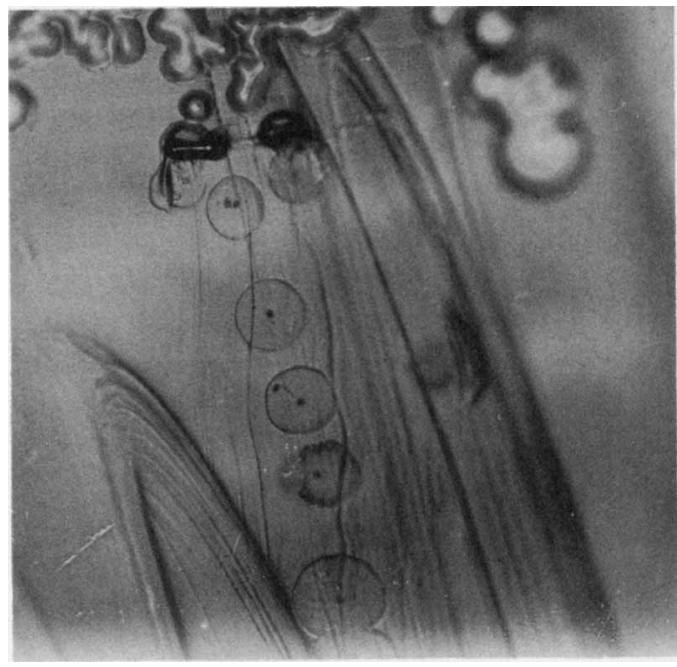

Fig. 3. Blood agar, 4 days. Corrosion marks on agar surface after removal of type 1 colonies. Magnification, $\times 4$.

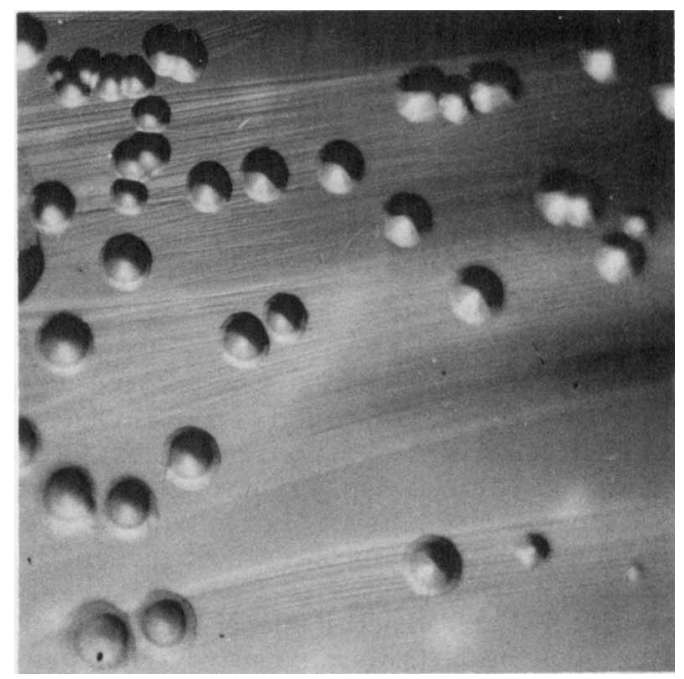

FIG. 4. Blood agar, 4 days. Round, smooth colonies (type 2) Magnification $\times 4$. 


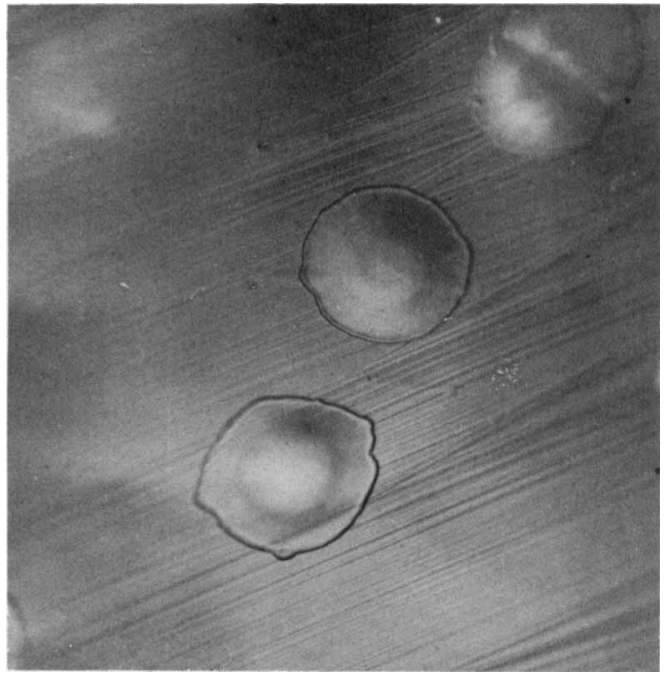

Fig. 5. Blood agar, 4 days. Large, flat, noncorroding colony derived from type 2 (colony type 4). Magnification $\times 4$.

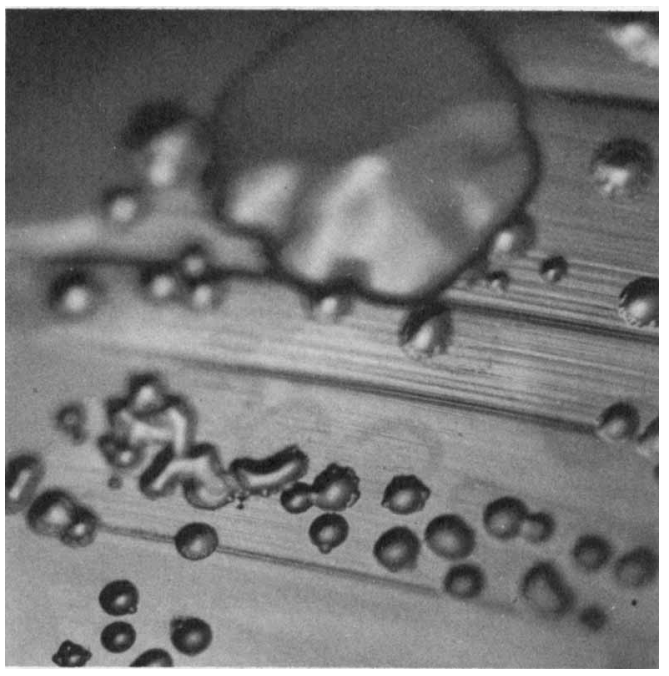

FIG. 6. Blood agar, 8 days. Colonies with thin, granular, spreading zones surrounding large colony. Magnification, $\times 5$.

tose. The oxidase reaction was positive with dimethyl- and tetramethyl-p-phenylenediamine. The catalase reaction, carried out on an agar slant culture, was very strongly positive. Nitrate was not reduced, but nitrite was reduced without the appearance of gas in the Durham tube. The $\mathrm{H}_{2} \mathrm{~S}$ reaction was weakly positive by the lead acetate paper-strip method. The following tests were negative: indole, citrate, phenylalanine and tryptophane deami- nase, and liquefaction of gelatin and coagulated serum.

Poly- $\beta$-hydroxybutyrate inclusions were not found in cells grown in Hutner medium (5) with $\beta$-hydroxybutyrate or in ordinary media.

Antibiotic susceptibility. The strain was strongly susceptible to penicillin, streptomycin, chloramphenicol, tetracyclines, and erythromycin.

Transformation of streptomycin resistance. Strain $6171 / 75$ was transformed nearly as efficiently by DNA from $N$. elongata as by autologous DNA, and vice versa (Table 1). Crosstransformation with $N$ meningitidis showed that the heterologous transformation both ways produced $23 \%$ of the number of transformants produced by autologous DNA. Strain 6171/75 was not transformed by DNA from $N$. catarrhalis and $M$. nonliquefaciens, nor were the latter two species transformed by DNA from strain $6171 / 75$.

\section{DISCUSSION}

The physiological and biochemical reactions of strain $6171 / 75$ suggest that it belongs to the genus Neisseria. This is supported by the results of transformation experiments, which reveal extremely close affinities between this strain and $N$. elongata strain M2 and strong compatibilities also with $N$. meningitidis.

Morphologically, and in most other characters, the strain showed very marked resemblance to $N$. elongata, so close that one might think that the strain represents a biotype of that species. The difference between the two, however (acid production from glucose, the catalase reaction, which is negative in most strains of $N$. elongata $[1,4]$ and strongly positive in strain $6171 / 75$, and the very marked differences in the consistency of the colonies: clay-like, coherent, and hydrophobic in N. elongata; and soft, homogenous, and easily emulsifiable in strain 6171/75) are more striking than the differences between some of the named species of Neisseria. If similar principles were to be followed in the classification of this strain as have been used previously in this genus, we feel that the strain would represent a new species. However, in view of the very close genetic relationship between this strain and $N$. elongata, we classify it as a subspecies of $N$. elongata. We propose the name Neisseria elongata subsp. glycolytica (Gr. n. gleukos sweet wine; Gr. adj. lyticus dissolving; M. L. adj. glycolyticus glucose-splitting) for this new subspecies. The type strain, $6171 / 75$, has been deposited in the National Collection of Type Cultures, Colindale, London (accession number, NCTC 
TABLE 1. Transformation of streptomycin resistance between strains $6171 / 75, N$. elongata M2, and $N$. meningitidis $M 1$

\begin{tabular}{llll}
\hline Recipient strain (colony count/ml) & \multicolumn{1}{c}{ Donor strain } & $\begin{array}{c}\text { Transformants/ } \\
\mathrm{ml}\end{array}$ & $\begin{array}{c}\text { Ratio of interstrain } \\
\text { to autologous } \\
\text { transformation }\end{array}$ \\
\hline Strain $6171 / 75\left(3.3 \times 10^{9}\right)$ & N. elongata M2 & $1.4 \times 10^{5}$ & $5.0 \times 10^{-1}$ \\
& N. meningitidis M1 & $9.1 \times 10^{3}$ & $3.3 \times 10^{-2}$ \\
& Strain $6171 / 75$ & $2.8 \times 10^{5}$ & \\
N. elongata M2 $\left(9.4 \times 10^{7}\right)$ & Strain $6171 / 75$ & $1.7 \times 10^{4}$ & $3.9 \times 10^{-1}$ \\
& N. meningitidis M1 & $3.4 \times 10^{2}$ & $7.7 \times 10^{-3}$ \\
& N. elongata M2 & $4.4 \times 10^{4}$ & \\
N. meningitidis $\mathrm{M} 1\left(4.7 \times 10^{8}\right)$ & Strain $6171 / 75$ & $5.9 \times 10^{4}$ & $1.8 \times 10^{-2}$ \\
& N. elongata M2 & $4.4 \times 10^{4}$ & $1.4 \times 10^{-2}$ \\
\hline
\end{tabular}

11050) and in the American Type Culture Collection, Rockville, Md. (accession number, ATCC 29315).

The subspecies appears to be a missing piece in the "Neisseria puzzle." The known species include saccharolytic and asaccharolytic cocci and an asaccharolytic rod-shaped species, and the existence of a saccharolytic rod-shaped organism is not surprising.

The pattern of colony variation in this strain resembles that found in several Moraxella and Neisseria species. Thus, the type 1 colony of this strain may be analogous to the SC type of colony of Moraxella species (3) or to the type 1 colony of $N$. gonorrhoeae, and the type 2 colony may be analogous to the normal $(\mathrm{N})$ colony type of Moraxella.

Objections may be raised against the establishment of a new subspecies based on only a single strain, but previous experience with $N$. elongata indicates that this may not always be inadvisable. The establishment of that species was on the basis of a single strain, but it was very quickly confirmed by several investigators $(1,4,7)$, and it may be anticipated that the description of this strain will incite others to report similar findings.

Further studies of this strain comparing it with other members of the family Neisseriaceae are in progress, and they will be published elsewhere.

\section{REPRINT REQUESTS}

Address reprint requests to: Dr. S. D. Henriksen, Kaptein W. Wilhelmsen og Frues Bakteriologisk Institutt, Rikshospitalet, Oslo 1, Norway.

\section{LITERATURE CITED}

1. Berger, U., and H. D. Piotrowski. 1974. Die biochemische Diagnose von Neisseria elongata. Med. Microbiol. Immunol. 159:309-316.
2. Bøvre, K. 1964. Studies on transformation in Moraxella and organisms assumed to be related to Moraxella. 1. A method for quantitative transformation in Moraxella and Neisseria, with streptomycin resistance as the genetic marker. Acta Pathol. Microbiol. Scand. 61:457-473.

3. Bøvre, K., T. Bergan, and L. O. Froholm. 1970. Electron microscopical and serological characteristics associated with colony type in Moraxella nonliquefaciens. Acta Pathol. Microbiol. Scand. Sect. B 78:765779 .

4. Bøvre, K., J. E. Fuglesang, and S. D. Henriksen. 1972 Neisseria elongata. Presentation of new isolates. Acta Pathol. Microbiol. Scand. Sect. B 80:919-922.

5. Bøvre, K., and S. D. Henriksen. 1976. Minimal standards for description of new taxa within the genera Moraxella and Acinetobacter: proposal by the Subcommittee on Moraxella and Allied Bacteria. Int. J. Syst. Bacteriol. 26:92-96.

6. Bovre, K., and E. Holten. 1970. Neisseria elongata sp.nov., a rod-shaped member of the genus Neisseria. Re-evaluation of cell shape as a criterion in classification. J. Gen. Microbiol. 60:67-75.

7. Hansen, W., E. Schoutens, and E. Yourassowsky. 1975. Isolément de souches microbiennes peu connues et recemment decrites: Neisseria elongata et Moraxella urethralis. Ann. Microbiol. Inst. Pasteur 126A:401-404.

8. Henriksen, S. D. 1969. Corroding bacteria from the respiratory tract. I. Moraxella kingii. Acta Pathol. Microbiol. Scand. 75:85-90.

9. Henriksen, S. D. 1974. "Pitting" and "corrosion" of the surface of agar cultures by colonies of some bacteria from the respiratory tract. Acta Pathol. Microbiol. Scand. Sect. B 82:48-52.

10. Henriksen, S. D., and K. Bøvre. 1969. Corroding and spreading colonies in Moraxella nonliquefaciens. Acta Pathol. Microbiol. Scand. Sect. B 76:459-463.

11. Henriksen, S. D., and J. Henrichsen. 1975. Twitching motility and possession of polar fimbriae in spreading Streptococcus sanguis isolated from the human throat. Acta Pathol. Microbiol. Scand. Sect. B 83:133140 .

12. Jyssum, K., and S. Lie. 1965. Genetic factors determining competence in transformation of Neisseria meningitidis. 1. A permanent loss of competence. Acta $\mathrm{Pa}$ thol. Microbiol. Scand. 63:306-316.

13. Lie, S. 1965 . Studies on the streptomycin resistance system of Neisseria meningitidis. Acta Pathol. Microbiol. Scand. 63:623-635. 